\title{
The prevalence of symptomatic sexually transmitted diseases and human immunodeficiency virus infection in outpatients in Lilongwe, Malawi
}

\author{
Johannes K Kristensen
}

\begin{abstract}
In June 1989 a pilot study on the prevalence of STDs was performed at Kamuzu Central Hospital, Lilongwe, Malawi. Among unselected out-patients an STD prevalence of $4.4 \%$ was found. The patients were predominantly males $(82 \cdot 7 \%)$, the age was predominantly $20-35 \mathrm{yr}$. The distribution of the diagnoses was dominated by a relatively large proportion of "ulcer-diseases" (syphilis, chancroid and lymphogranuloma venereum) making up $67 \%$ of the patients. Almost two thirds of the patients were HIV-positive $(62 \cdot 4 \%)$. No significant difference was found in HIV-infection prevalence when patients with ulcer diseases were compared with patients with discharges. Among the patients with gonorrhoea a prevalence of penicillinase-producing Neisseria gonorrhoeae of $53 \%$ was found. It is concluded that a plan for the management of STDs is urgently needed as it is of paramount importance to combat STDs in order to prevent the spread of HIV-infection.
\end{abstract}

\section{Introduction}

The extent of the STD problem in Malawi is not known. With the emergence of the AIDS epidemic and the known inter-relationship between STDs and AIDS it has become important to formulate a more comprehensive plan for cure of, education in and information about STDs.

In the central region of Malawi, including the capital Lilongwe, about 10000 cases of STD were diagnosed and reported in 1987. This is a case rate of 328 per 10000 . At present little is known about the individual diseases in the group of STDs. AIDS is a heterosexual disease in Africa and the sex-ratio has been found to be $1 \cdot 1: 1{ }^{1}$ The most important riskgroups are: prostitutes, individuals with several sex-

Kamuzu Central Hospital, Lilongwe, Malawi and Department of Dermatology and Venereology, Bispebjerg Hospital, Copenhagen, Denmark

Johannes K Kristensen partners, patients with a history of genital sores and other STDs and finally recipients of blood. In Nairobi, Kenya, $60 \%$ of men with chancroid reported contact with prostitutes as a source of the infection. ${ }^{2}$

Sero-surveillance in antenatal clinics is regarded as the most accurate indicator of HIV prevalence in the general population of women of child-bearing age. The incidence in this particular group has in Malawi been shown to increase from $4.2 \%$ in 1986 to $17.4 \%$ in 1988. This increase has been especially marked in the urban areas. ${ }^{3}$

By the middle of 1988 over $10 \%$ of all patients admitted to medical wards of Zomba General Hospital in Southern Malawi had illnesses related to HIV-infection. ${ }^{4}$ In blood donors at Queen Elisabeth Central Hospital, Blantyre (Southern region), the prevalence oscillated between $15 \%$ and $30 \%$ in 1988 , while in Lilongwe at Kamuzu Central Hospital (Central region) the prevalence rate was $8-18 \%$ in the same year. ${ }^{3}$

Penicillin resistant Neisseria gonorrhoeae (PPNG) was reported from Africa as early as 1976 . Since then the occurrence of penicillin-resistant strains (PPNGs as well as chromosome-linked resistance) has increased considerably. PPNGs have been found with the following percentages in Southern and East Africa: Malawi $71 \%$, Uganda $60 \%$, Tanzania $50 \%$, Kenya $50 \%$, Zimbabwe $40.8 \%$, Botswana rural areas $28 \%{ }^{5}$ It has been reported from Gabon that the prevalence of PPNG strains were $50 \%$ in 1985 . After the introduction of spectinomycin the prevalence has dropped to $30 \% .^{5}$

\section{Study objectives}

It was decided to perform a study on the prevalence of STDs in Lilongwe with the following objectives:

(1) To determine the prevalence of symptomatic STDs in a general out-patient clinic.

(2) To evaluate HIV-status in STD patients.

(3) To evaluate the incidence of drug-resistance in gonorrhoea.

This study was approved by the Malawi Medical Research Council.

Methods

For the purpose of this study, Kamuzu Central 
Hospital in Lilongwe was chosen with a recording period of 4 weeks, as the sample size was expected to be $700-800$. The study took place at a general outpatient department. Only adults ( $>12 \mathrm{yr}$ ) were seen as children attended the "under five clinic". The patients were unselected and attended on their own accord.

The catchment area of the clinic was known as it was shown that $90 \%$ of the patients lived within a distance of 15 kilometres. It was planned that all patients who complained of urethral or vaginal discharge should be examined by gonococcal culture (and sensitivity testing if culture-positive). All adult patients reporting to the out-patient department were interviewed by Clinical Officers, who also performed the clinical examination supervised by the study-coordinator.

HIV-ELISA was performed in all patients who by interview and objective examination were found to be suffering from an STD. All laboratory examinations were performed confidentially as only a recording number was known by the laboratory.

Three Clinical Officers were employed as datacollectors and a nurse was employed for blood sampling and linking with the laboratory, where two laboratory technicians with a special interest in the study were performing culture and sensitivity testing and serology.

The VDRL reaction was performed in all patients using a routine technique. HIV-antibodies were detected by an ELISA-technique (Dupont De Nemours, Geneva). Borderline cases were confirmed by Western blot (Dupont De Nemours, Geneva). Small samples of tests were regularly confirmed by Western blot. Owing to shortage of HIV-ELISAkits only 580 patients were finally tested. These were chosen as random numbers from frozen serum samples.

Gonococcal culture was performed on modified New York City medium. Screening and confirmatory tests were performed as well as testing for beta-lactamase by an indicator-method. Only the first 173 patients with discharges were finally cultured because of lack of substrates.

The study-coordinator supervised data-collection to ensure a uniformity in diagnosis and treatment of the patients during the study period.

Recording and analysis of the data was performed using the WHO software EPI-INFO. In the statistical comparisons the Chi square and Fishers exact tests were used.

\section{Results}

A total of 16218 patients (aged $13 \mathrm{yr}$ and above) were screened during a period of 4 weeks. Of these 705 were classified as suffering from an STD. The prevalence rate was $4.4 \%$.

Male patients made up for $81.7 \%$ and females
Table 1 The main diagnostic groups

\begin{tabular}{lccc}
\hline & Male & Female & Total \\
\hline Ulcer disease & 395 & 87 & $482(68 \cdot 3)$ \\
Discharges & 175 & 40 & $215(30 \cdot 5)$ \\
HIV-associated disease & 4 & 2 & $6(0 \cdot 9)$ \\
Genital warts & 2 & 0 & $2(0 \cdot 3)$ \\
Total & $576(81 \cdot 7)$ & $129(18 \cdot 3)$ & $705(100)$ \\
\hline
\end{tabular}

Percentage in parentheses.

Table 2 Distribution of HIV reactivity in relation to the main diagnostic groups

\begin{tabular}{lll}
\hline Diagnosis & No tests & No reactive \\
\hline Ulcer disease & 398 & $258(64 \cdot 8)$ \\
Discharges & 182 & $104(57 \cdot 1)$ \\
Total & 580 & $362(62 \cdot 4)$ \\
\hline
\end{tabular}

Percentage in parentheses.

$18 \cdot 3^{\circ}{ }_{0}$. This was significantly different from the overall sex-ratio which was $55 / 45 \%(p<0.001)$.

The mean age was $26.8(\mathrm{SD}=7.5)$. Of the patients 365 were married $(56.6 \%)$ while 302 were single $\left(42 \cdot 9^{\circ}{ }_{0}\right)$.

The main diagnostic groups can be seen from table 1. Some overlap between groups was experienced, as $3{ }^{\circ}{ }_{0}$ of ulcer patients had a discharge and $5 \%$ of discharge-patients also had a genital ulcer. The distribution of the diagnoses was not significantly different in the female patients $(p>0.9)$.

The VDRL was positive (titre 2 to 128 ) in $7 \%$ of patients with ulcers and $1 \%$ of patients with discharges $(p<0.001)$. In table 2 the distribution of HIV-reactivity in relation to the main clinical diagnosis is shown. Twenty samples were borderline in HIV-ELISA but were confirmed by Western blot. Although 11 were still borderline in Western blot they were grouped with the positive results. No significant difference between patients with ulcer diseases or patients with urethral or vaginal discharge was found ( $p=0.09$, Fishers exact test). The HIVreactivity pattern was not different in the females.

In six cases ARC (Aids Related Complex) was diagnosed clinically at the first visit in patients suffering from diarrhoea, weight loss and general lymphadenopathy. All six were HIV-positive. Gonococcal culture was performed in cases where urethral or vaginal discharge was a clinical finding. In the first 173 consecutive patients where discharge was the main finding, culture was performed and found positive in $113(65 \%)$. The beta-lactamasetesting gave as a result that 70 strains $(53 \%)$ were PPNG-strains while 62 strains $(47 \%)$ were PPNGnegative.

\section{Discussion and conclusions}

The prevalence of STDs is relatively high in Malawi, 
as judged from available statistics. As little was known concerning the prevalence rate of ulcers versus discharges it was decided to perform a pilotstudy with the aim also of obtaining data on HIVreactivity and drug-resistance in STD patients. In the largest outpatient department in Lilongwe the prevalence of STDs was found to be $4.4 \%$ in a cluster of unselected patients. This figure was very close to the reported national average in OPDs. The patients were predominantly males, which might be related to a reservoir of untreated females. The ratio of male to female patients was found to be significantly different from the whole population screened. The agedistribution showed the expected dominance of the sexually active agegroup between 20 and 35 years.

The distribution of the main diagnoses was as previously described for other tropical countries. ${ }^{6}$ The number of cases of ulcer diseases (syphilis, chancroid and lymphogranuloma) was relatively high. All diagnoses were based on clinical criteria only, according to Manson and Bell. ${ }^{6}$ The correct diagnosis will be missed even by experienced specialists in a huge proportion of cases if clinical criteria alone are available for the diagnosis. As a consequence it was chosen to present the results as syndromes and not as definite diagnostic groups. Asymptomatic carriage of STDs is also common especially in the female, but in this study it was possible only to identify symptomatic cases. The asymptomatic patients, as well as being at risk of developing complications themselves, constitute an important reservoir of infection which will have to be identified in the future.

Sexually transmitted pathogens now comprise a wide spectrum of medical microbiology. It follows that the diagnosis of most of the conditions, besides requiring a high degree of suspicion and careful examination by appropriately trained personnel, can be confirmed only by laboratory methods, necessitating proper collection of specimens, technical expertise and equipment. The present study was done with less than minimal equipment, as a dark-field microscope and facilities for culture of Haemophilus ducreyi and Chlamydia trachomatis were not available. A sensitive screening test for syphilis was also missing. The results of the VDRL reaction showed a surprisingly low number of reactive sera, even in the ulcer group, although reactivity in the ulcer group was significantly more common than in the discharge group. At present no information is available on VDRL reactivity in general non-STD out-patients.

The prevalence of HIV-reactivity almost reached two thirds of the patients, with no significant difference between patients with ulcer diseases or discharges. This finding might seem to contradict findings by other authors ${ }^{7}$ but it must be taken into account that a previous episode of "ulcer disease" is common in this patient-group.
The overall results of the present study are in accordance with a recent report from $R$ wanda, on the distribution of diagnosis in genital ulcers and on HIV reactivity. In that study overall $59 \%$ of the patients had IgG antibodies to HIV ${ }^{8}$ The prevalence of HIV reactivity in general out-patients is not known at present but in antenatal clinics in Malawi it amounted to $17.4 \%$ in 1988 . In blood-donors in Lilongwe HIV reactivity oscillated between $8 \%$ and $18 \%$ in the same year. ${ }^{3}$

The culture results in gonorrhoea showed a prevalence of PPNG-strains of $53 \%$ confirming previous studies from Malawi. ${ }^{5}$ The number of cases of STDs and the problems encountered in their management clearly makes it important as soon as possible to establish a plan for the management of STDs. It will help to prevent the spread of HIV infection if the incidence of these diseases could be reduced. ${ }^{9}$

STD treatment and control programmes, especially those aimed at prostitutes and heterosexually promiscous men, should serve as an important adjunct to health education campaigns and the promotion of condom use in retarding the rapid spread of the HIV epidemic. ${ }^{8}$

Genital ulcers are the most frequent STD in Malawi. As substantial evidence has been accumulated in Nairobi ${ }^{10}$ of the influence of this disease-group on HIV-transmission, future strategies must focus on the epidemiology of these particular diseases.

J A Kalilani, MD, Malawi Ministry of Health AIDSprogram is thanked for help during initiation of the study. O Brasseur, $M D$, WHO is thanked for advice on planning and data-processing.

1 Mann JM, Chin J, Piot P, Quinn T. The International epidemiology of AIDS. Sci Am 1988;259:60-9.

2 Simonsen JN, Cameron W, Gakinya MN, et al. Human immunodeficiency virus infection among men with sexually transmitted diseases. N Engl J Med 1988;319:274-8.

3 Malawi Ministry of Health AIDS-program-Proposal 1988

4 Reeve PA. HIV infection in patients admitted to a General Hospital in Malawi. Br Med J 1989;298:1567-8.

$5 \mathrm{Vth}$ African Regional Conference on Sexually transmitted diseases 1987.

6 Manson's tropical Diseases. Manson-Bahr PEC and Bell D, eds. London: Bailliere-Tindall 1987.

7 Kreiss J, Caraël M, Meheus A. Role of sexually transmitted diseases in transmission of human immunodeficiency virus. Genitourin Med 1988;64:1-2.

8 Bogaerts J, Ricart CA, van Dyck E, Piot P. The etiology of genital ulceration in Rwanda. Sex Transm Dis 1989;16:123-6.

9 Ngugi EN, Simonsen JN, Bosire M, et al. Prevention of transmission of human immunodeficiency virus in Africa: Effectiveness of condom promotion and health education among prostitutes. Lancet 1988;ii:887-90.

10 Ronald AR, Plummer F. Chancroid: A newly important sexually transmitted disease. Arch Dermatol 1989;125:1413-4.

Address for correspondence: Johannes K Kristensen, MD, Department of Dermatology, Bispebjerg Hospital, DK-2400 Copenhagen NV, Denmark.

Accepted for publication 4 May 1990 